\title{
Squamous cell cancer of the cervix: prognostic factors related to survival
}

\author{
M.P. HOPKINS \& G.W. MORLEY \\ Department of Obstetrics \& Gynecology, University of Michigan Medical Center, Ann Arbor, Michigan 31906, USA
}

\begin{abstract}
Hopkins MP, Morley GW. Squamous cell cancer of the cervix: prognostic factors related to survival. Int J Gynecol Cancer 1991; 1: 173-177.
\end{abstract}

Seven hundred and fifty-three patients with invasive squamous cell cancer of the cervix treated at the University of Michigan from 1970-1985 are reported. These included stage IA 43, stage IB 345, stage IIA 27, stage IIB 163, stage IIIA 4, stage IIIB 113, stage IVA 32, stage IVB 26. The age ranged from 18 to 92 years with a mean of 49.9 years. Clinical characteristics included: nulliparity $11 \%$, married $93 \%$, obese $41 \%$, hypertensive $37 \%$, diabetes $10 \%$, smoking $50 \%$, bleeding $76 \%$. The cumulative fiveyear survival for all patients was $67 \%$ and this was influenced by the stage of disease: stage IA $98 \%$, stage IB $89 \%$, stage IIA $72 \%$, stage IIB $62 \%$, stage III $37 \%$, stage IVA $14 \%$, stage IVB $4 \%$. Patients with a welldifferentiated tumor had an $85 \%$ survival rate while those with a poorly differentiated tumor had a $57 \%$ survival rate. The probability of metastatic disease to lymph nodes corresponded to the stage of disease; stage I $17 \%$, stage II $55 \%$, stage III $70 \%$, stage IV $81 \%$. When lymph nodes were negative, the survival rate for all patients was $86 \%$ while those with positive nodes had a $33 \%$ survival rate. Factors which influenced survival in the univariate analysis included stage, node status, tumor grade, age, interval from previous pelvic examination, diabetes. Only stage, node status and tumor grade maintained significance in the multiple proportion hazard analysis.

KEYWORDS: cancer cervix, squamous cell cancer.

Carcinoma of the cervix continues to be a leading cause of morbidity and mortality among gynecologic malignancies. Despite the institution of widespread cytologic screening, this remains the third most common gynecologic malignancy diagnosed and the second leading cause of death among gynecologic malignancies in the USA. Among carcinomas of the cervix, squamous cell cancer remains the most common pathologic type. Authors have reported a number of factors that influence the prognosis in squamous cell cancer of the cervix. These include age, diabetes, smoking, parity, anemia, and extent of

Address for correspondence: Michael P. Hopkins, MD, Associate Professor, Department of Obstetrics \& Gynecology, Northeastern Ohio Universities, College of Medicine, 400 Wabash Avenue - A.C.C. Akron, Ohio 44307, USA. disease ${ }^{(1-5)}$. This study was undertaken to evaluate various prognostic factors related to survival for squamous cell carcinoma of the cervix.

\section{Material and methods}

The clinical records, pathology reports, and tumor conference notes were reviewed for patients registered at the University of Michigan Medical Center (UMMC) with a diagnosis of carcinoma of the cervix between 1970 and 1985. All patients having squamous cell carcinoma of the cervix were reviewed. Information was recorded for the clinical presentation, medical history, height, weight, oral contraceptive usage, treatment modality, tumor grade, and size of the cervical lesion. All patients were staged according to the 
International Federation of Gynecology and Obstetrics (FIGO) guidelines. We defined microinvasive carcinoma (stage IA) where invasion did not exceed $3 \mathrm{~mm}$ and/or angiolymphatic invasion was not present. Patients with tumors that extended just into the parametrium were considered to have small stage IIB tumors while those that extended close, but not to the side wall, were considered to have large stage IIB tumors. Patients underwent staging by the gynecologic oncologist and the radiation oncologist, with discussion at the Gynecologic Tumor Conference. Patients with stage I disease routinely underwent IVP and chest $X$-ray. Patients with more advanced disease routinely underwent chest $X$-ray, IVP, cystoscopy, barium enema, and sigmoidoscopy. Selected patients underwent lymphangiogram and in the later years CAT scanning was routinely done. Radiation therapy during this time period was given with external beam and brachytherapy to give a combined total dose of $8000-9000$ Cgy to point A. Therapy utilized the split field technique where 1980 Cgy (180 Cgy per day) of whole pelvic radiation was given. This was followed by two intracavitary applications to a total of 7-8000 $\mathrm{mg} \mathrm{h}^{-1}$. External beam with a midline block completed the therapy bringing the pelvic sidewall to $5000 \mathrm{Cgy}$.

The original hematoxylin and eosin pathology slides were reviewed. When slides were unavailable, the microscopic features were recorded according to the original pathology report and review from the Gynecologic Tumor Conference. Well-differentiated tumors were defined when there was little nuclear pleomorphism and the cytologic atypia was not pronounced. Cells were generally large with abundant cytoplasm and a more normal nuclear to cytoplasmic ratio. In general the well-differentiated tumors produced keratin, and mitotic figures were infrequent. Poorly differentiated tumors had pronounced nuclear pleomorphism, hyperchromatic nuclei and scant cytoplasm. Cells were smaller, mitoses were frequent and keratin formation was usually absent. Moderately differentiated tumors had intermediate characteristics. Patients with advanced lesions where brachytherapy could not be performed, received only external beam radiotherapy, 6500-7000 Cgy. All external beam therapy was given with cobalt 60 until 1981 and then by linear accelerator (Clinac 18, 10-MV photons). Hysterectomy after radiation was not utilized.

Statistical analysis was performed by the University of Michigan, Department of Biostatistics, School of Public Health. Survival was plotted with the life table method described by Kaplan \& Meier ${ }^{(6)}$. The generalized Savage (Mantel-Cox) method was used to test differences in survival ${ }^{(7-8)}$. A Cox Model stepwise proportional hazard analysis was performed to compare the hazard for tumor-related deaths to potential risk factors. A two-way cross tabulation with $\chi^{2}$ analysis was used to evaluate the various clinical features in relation to the stage of disease.

\section{Results}

During the time period 1970-1985, 753 patients with invasive squamous cell carcinoma of the cervix were treated. The number of patients by each stage included: stage IA $43(5.7 \%)$; stage IB $345(45.8 \%)$; stage IIA $27(3.5 \%)$; stage IIB $163(21.7 \%)$; stage IIIA $4(0.2 \%)$; stage IIIB $113(15.3 \%)$; stage IVA $32(4.3 \%)$; stage IVB $26(3.5 \%)$. The age at time of diagnosis ranged from 18 to 95 years with a mean age of 49.9 years. The mean age and range by stage was stage IA 39 years (21-75 years), stage IB 44.6 years (18-89 years), stage II 55 years (25-93 years), stage III 56 years (25-92 years), stage IV 60.5 years (34-95 years). Clinical characteristics included: multiparity $89 \%(674 / 756)$; married $93 \%$ (699/753); obese $41 \%$ (306/752); hypertensive $37 \%$ (281/752); diabetes $10 \%$ (76/752); oral contraceptive usage at the time of diagnosis for patients less than 45 years old $26 \%$ (76/297); smoking 50\% (352/699); presenting symptoms of bleeding $76 \%$ (567/748); positive cytology at the time of diagnosis $82 \%(432 / 527)$. Three patients received the radiation sensitizer hydroxyurea.

The node status was significantly influenced by the stage of disease. When the node status was available, the following percentages of positive nodes were found: stage I $17 \%$ (45/261); stage II $55 \%$ (43/78); stage III $70 \%(46 / 66)$; stage IV $81 \%(39 / 48)$. The presence of bilateral node metastases significantly $(p=0.00001)$ increased with the stage of disease: stage I $7 \%(19 / 261)$; stage II $27 \%$ (21/78); stage III $45 \%$ (30/66); stage IV $71 \%$ (34/48). Clinical features that were significantly related to an advancing stage of disease included tumor grade, age of the patient over 60 years of age, obesity, hypertension, diabetes, symptom of bleeding, interval from cytologic examination. Factors which were not related to the stage of disease included parity, marital status, oral contraceptive usage less than 45 years old, smoking and positive cytology at the time of diagnosis.

The cumulative five-year survival rate for all patients with invasive squamous cell carcinoma excluding microinvasion was $67 \%$ (Fig. 1). Survival rate was significantly influenced by stage (Fig. 2), stage IA $98 \%$, stage IB $89 \%$, stage IIA $72 \%$, stage IIB $62 \%$, stage 


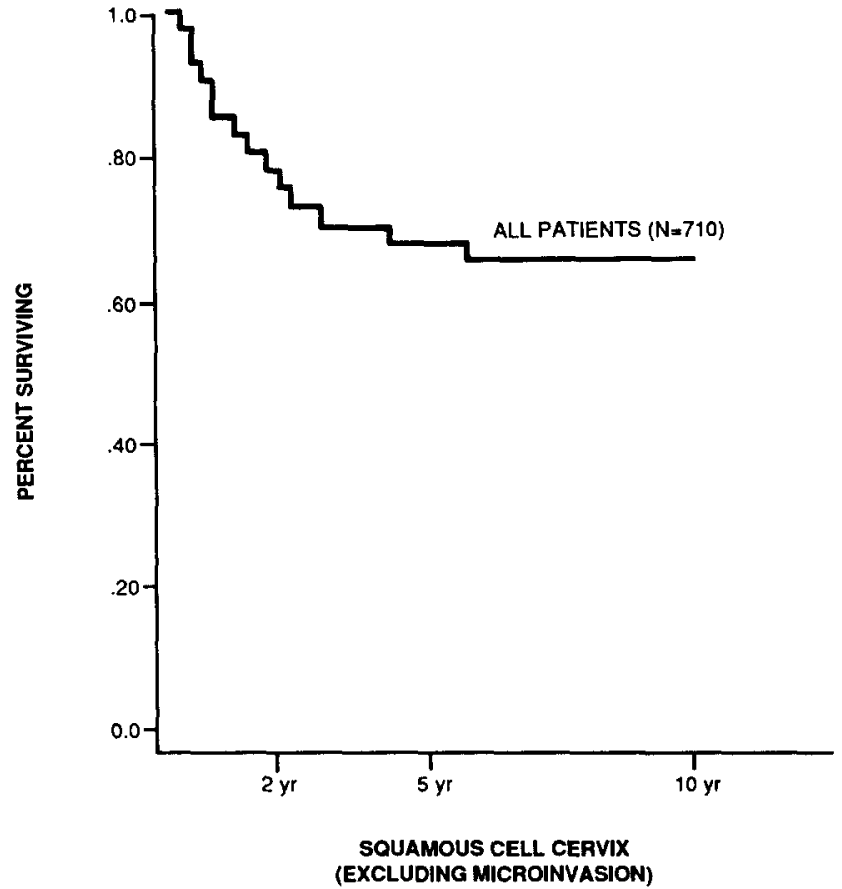

Fig. 1. Overall survival for patients with invasive squamous cell cancer of the cervix.

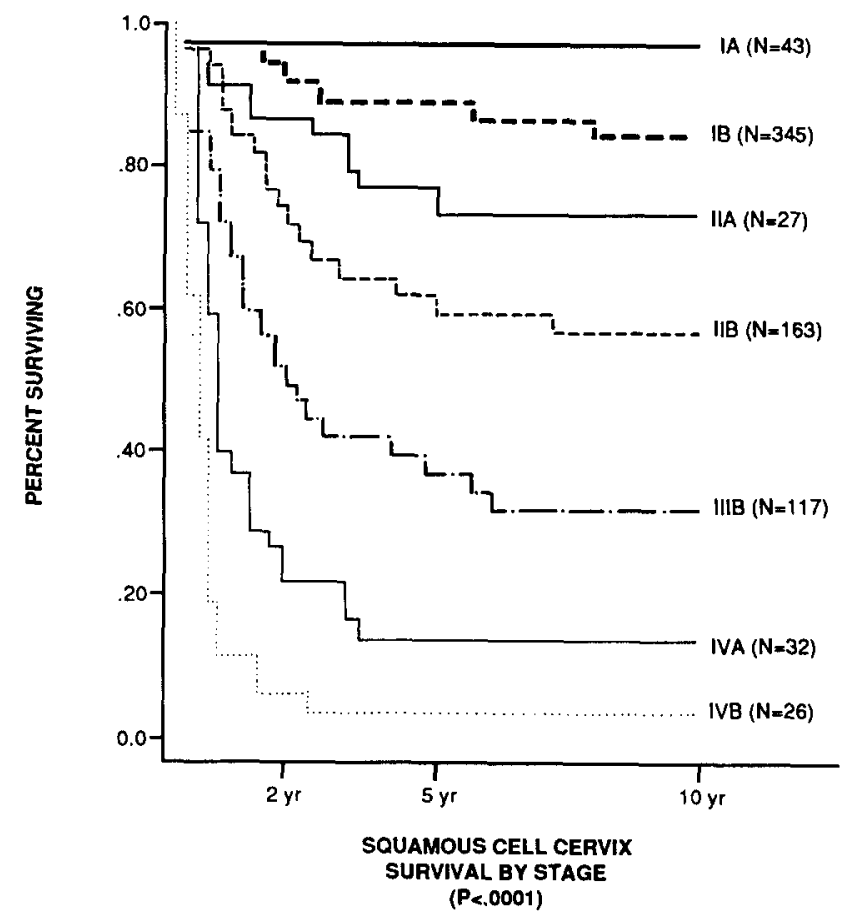

Fig. 2. Survival by stage of disease for patients with invasive squamous cell cancer of the cervix.

III $36 \%$, stage IVA $14 \%$, stage IVB $4 \%$. Survival rate was influenced significantly by the tumor grade (Fig. 3). Patients with well-differentiated tumor had an $85 \%$ cumulative five-year survival rate decreasing to $57 \%$

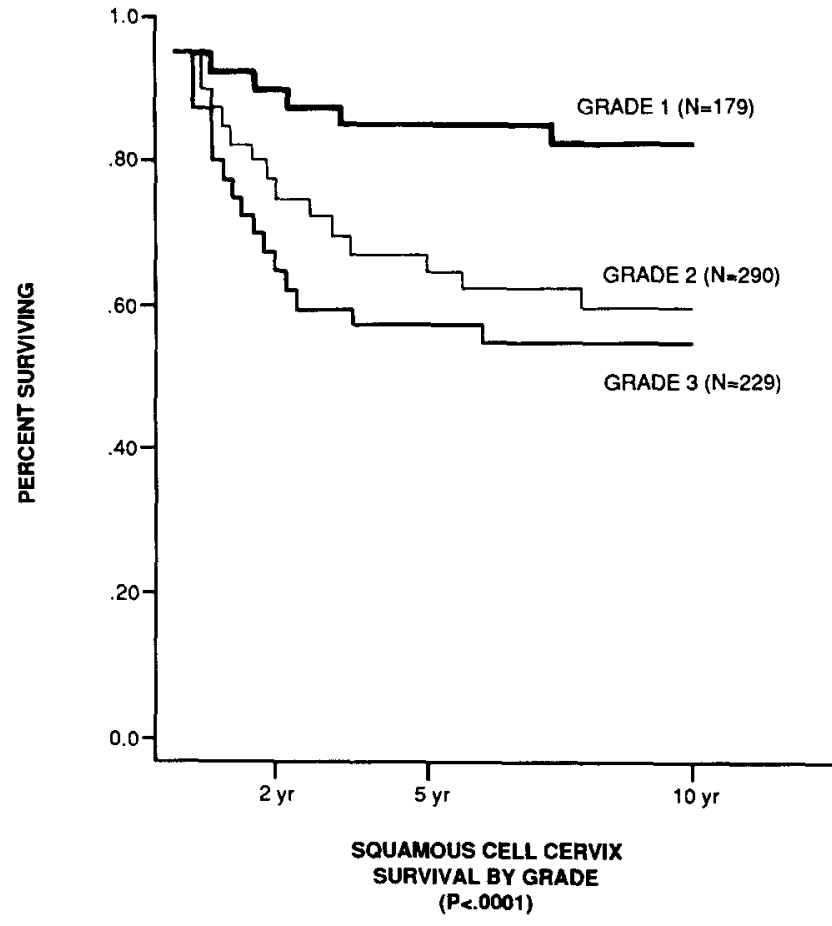

Fig. 3. Survival by tumor differentiation for all patients with invasive squamous cell cancer of the cervix.

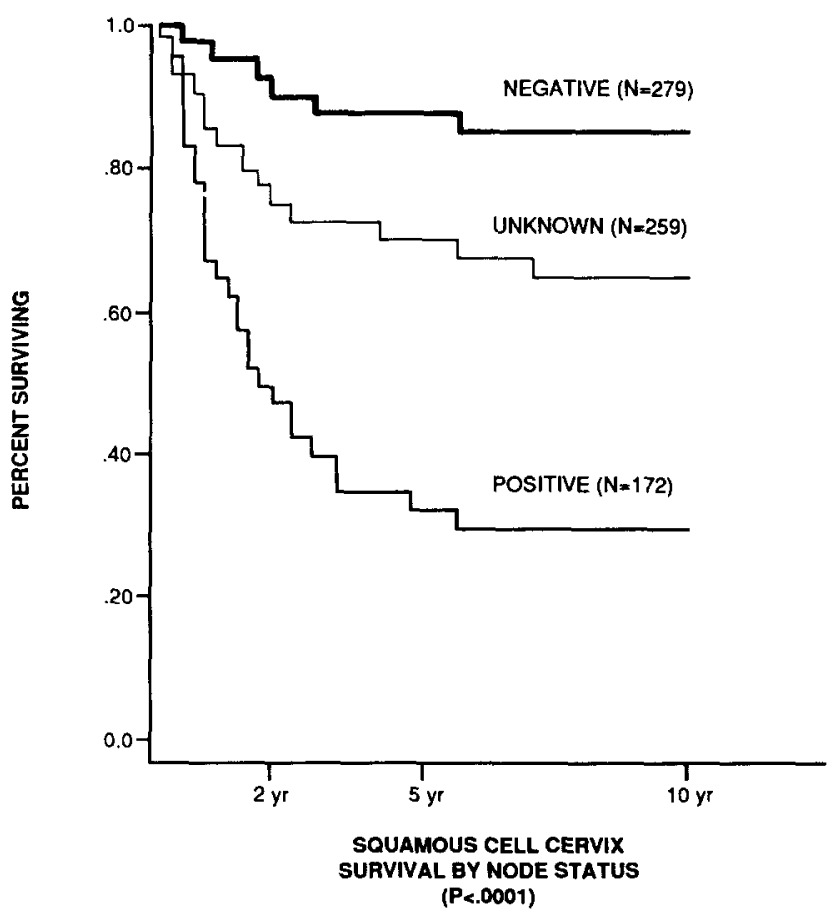

Fig. 4. Survival according to the presence or absence of metastatic disease to the lymph nodes for all patients with invasive squamous cell cancer of the cervix.

for those with a poorly differentiated tumor. The presence of metastatic disease to the lymph nodes significantly influenced survival (Fig. 4). There was an $86 \%$ 


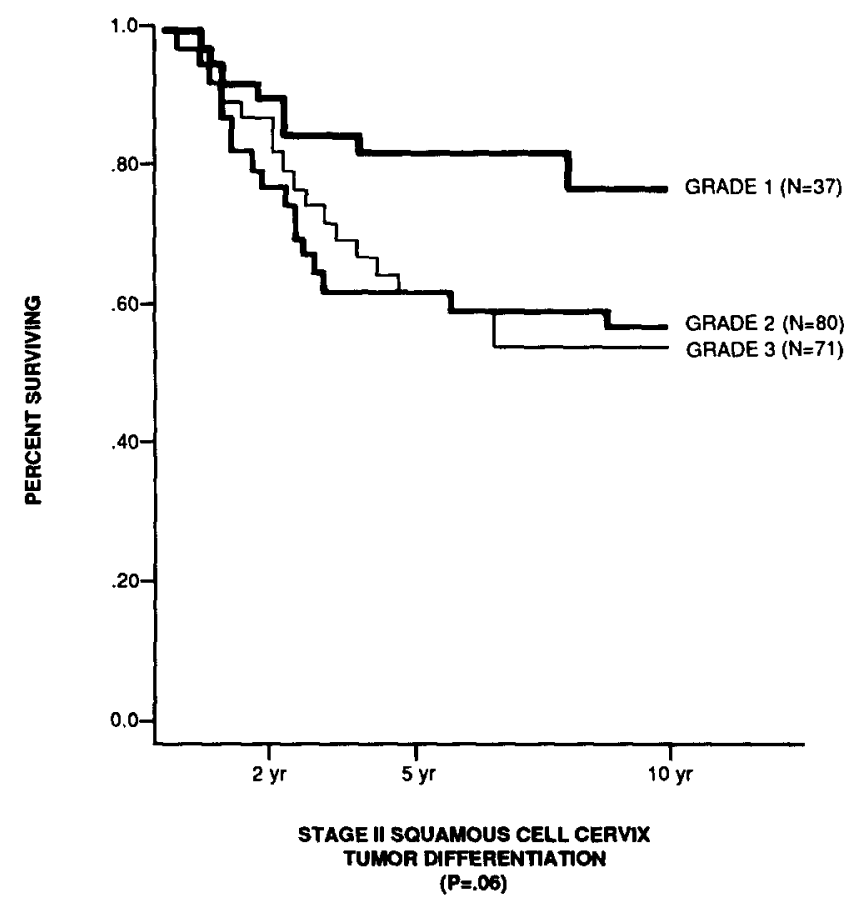

Fig. 5. Survival by tumor differentiation for patients with stage II invasive squamous cell cancer of the cervix.

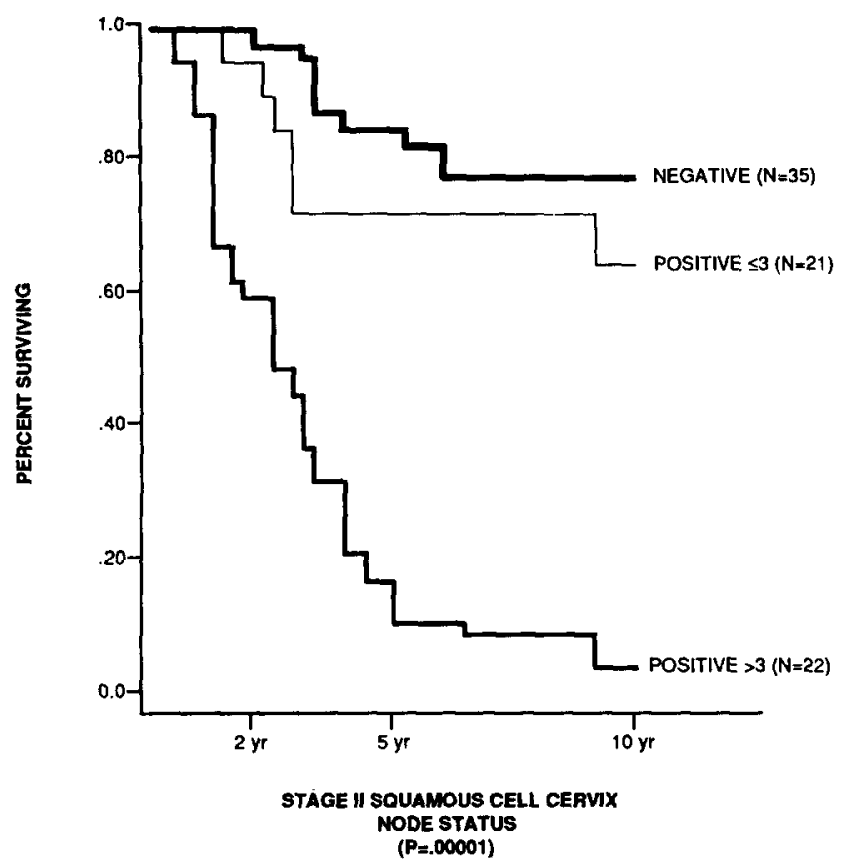

Fig. 6. Survival according to the presence or absence of metastatic disease to lymph nodes for stage II.

survival rate for those with negative lymph nodes compared to $33 \%$ for those with positive lymph nodes. The interval from the previous cytology significantly influenced survival. Those who had a pelvic examination with cytology performed within one year had a $78 \%$ survival rate compared to $60 \%$ for those who had gone more than three years $(p=0.0001)$. The patients with diabetes had a $50 \%$ five-year survival rate compared to those without diabetes $(p=0.0005)$. Smoking did not significantly influence survival. In the Cox Model multiple proportion hazard analysis the significant predictors of survival included stage, lymph node status and grade of tumor. Factors found not to predict survival when other factors were incorporated included diabetes, previous cytology, age and smoking history.

A separate analysis was performed for patients with stage II disease. There was no significant difference in survival between stages IIA and IIB, and both groups were combined for further analysis. All patients with stage II had a combined cumulative five-year survival rate of $61 \%$. The extension of disease did not significantly influence survival. Those with large tumors almost to the side wall had a $61 \%$ survival rate while those with tumors of smaller size had a $67 \%$ survival rate. The tumor differentiation was significant. Those with well-differentiated tumors had an $83 \%$ cumulative five-year survival rate while those with moderate and poorly differentiated tumors had a $59 \%$ survival rate (Fig. 5). Regarding the stage II patients, the node status significantly influenced survival. Those with negative nodes had a $79 \%$ five-year survival rate. Those with three or fewer positive nodes had a $74 \%$ survival rate while those with more than three positive nodes had a $9 \%$ survival rate (Fig. 6 ). The status of the paraaortic nodes significantly influenced survival for those patients with positive pelvic nodes. When paraaortic nodes were negative, the cumulative fiveyear survival rate was $57 \%$, and survival decreased to $6 \%$ when these were positive (Fig. 7). During this time period, radiation therapy was not routinely given to the paraaortic area. Other factors that did not significantly influence survival in stage II disease included age, diabetes, hypertension and obesity.

Patients who underwent radiation therapy and failed with central and/or regional disease included: stage IB $1(1 \%)$; stage IIB $4(2.4 \%)$; stage IIIB $7(6 \%)$. One patient with stage IB disease and one patient with stage IIB disease developed central failure after primary radiation therapy and underwent pelvic exenteration. Both are free of disease. Two patients with stage IIIB disease underwent pelvic exenteration for central failure and both died of disease. Seven patients $(3.2 \%)$ failed after radical hysterectomy. There were five patients with only a cuff recurrence and two were salvaged by radiation therapy. One of these five patients underwent a pelvic exenteration after salvage radiation therapy and died of disease.

Major complications requiring hospitalization or 


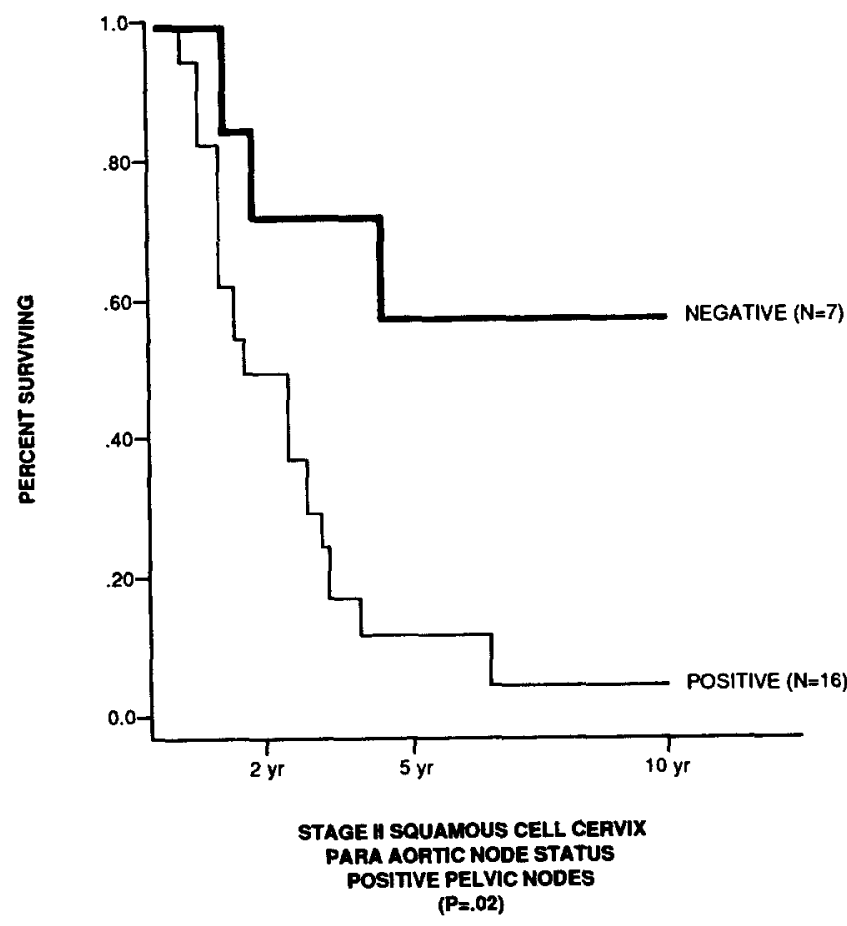

Fig. 7. Survival according to the presence or absence of metastatic disease to the paraaortic lymph nodes for patients with stage II disease and positive pelvic lymph nodes.

treatment delay are listed in Table 1. Significant complications not listed included pyometra 8 , and cystitis 2 . The fistula rate for stage I disease was $2.3 \%$ among patients treated by radical hysterectomy, and $1 \%$ for

Table 1. Major radiation therapy complications by stage of disease and total for all stage I-III patients. (E/P-enteritis/ proctitis; SBO-small bowel obstruction; Fist-fistula). (Stage I patients treated by radiation only)

\begin{tabular}{lrrrrr}
\hline Stage & E/P & SBO & Fist & No. & $\%$ \\
\hline I & 6 & 7 & 1 & $14 / 111$ & 12.6 \\
II & 4 & 3 & 7 & $14 / 163$ & 8.5 \\
III & 3 & 2 & 13 & $18 / 117$ & 15.4 \\
Total & 13 & 12 & 21 & $46 / 391$ & 11.7 \\
\hline
\end{tabular}

Table 2. Fistula complications for patients treated with radiation therapy by stage of disease. (VV-vesicovaginal; RV-rectovaginal; Combined - both recto and vesicovaginal) ${ }^{*} 8$ patients had fistula at presention

\begin{tabular}{lccccc}
\hline Stage & VV & RV & Combined & No. & $\%$ \\
\hline I & & 1 & & $1 / 111$ & 1 \\
II & 1 & 3 & 3 & $7 / 163$ & 4.2 \\
III & 2 & 7 & 4 & $13 / 117$ & 11.1 \\
IV & 7 & & 2 & $9^{*} / 32$ & 28 \\
\hline
\end{tabular}

those treated with radiation therapy. The vaginal fistulas by stage among patients treated by radiation are listed in Table 2.

\section{Discussion}

The results reported here reaffirm the prognostic value of clinical staging for squamous cell carcinoma of the cervix. Recently surgical staging for cervical cancer has been reported to be only of limited value due to the inability to control metastatic disease with our current therapy ${ }^{(9)}$. Thus clinical staging will maintain its importance and remain an important prognostic variable. The survival rate reported here is very similar to that reported by Johnson $e t$ al. ${ }^{(2)}$, with survival directly proportional to the stage of disease. In Johnson's report, $58 \%$ of patients had a central or side wall recurrence which is much higher than the percentage reported here. The overall central failure rate in our patients was $9 \%$ and only five underwent exenteration. When patients failed treatment, the vast majority of our patients had distant disease with isolated central disease playing only a limited role. The pelvic failure rate reported here is similar to that recently reported by Stehman ${ }^{(10)}$ from the GOG series utilizing the radiation sensitizer Hydroxyurea. In our group of patients, however, only a very small number received radiation sensitizers.

The influence of tumor grade in squamous cell cancer of the cervix has been reported to influence survival ${ }^{(1,4)}$. In this report the poorly differentiated squamous cell cancers had a decreased survival rate even when accounting for the stage in the multiple proportional hazard analysis. These results are similar to those of Prempree who reported a decrease in survival rate in stage I from $79 \%$ for grade one to $43 \%$ for grade three, and in stage II from $58 \%$ for grade one to $44 \%$ for grade three. Prempree also reported that the patients with poorly differentiated tumors had a higher percentage of metastatic disease ${ }^{(1)}$.

The lymph node status appears to be a critical determinant of survival. The survival is markedly decreased stage for stage when metastatic disease to the lymph node is present. Additionally, as tumor stage progresses there is a higher percentage of node metastases and a higher propensity for bilateral node metastases.

In our report a number of factors in the univariate analysis were significant for predicting survival. However, in the multiple proportion analysis, the factors that overwhelmingly influenced survival were the stage of disease, the lymph node status, and the tumor differentiation. Authors have reported the effects of 
other factors on survival. Kapp et al. ${ }^{(4)}$ utilizing a multiple proportion hazard analysis reported that increasing age, diabetes, and increasing parity also played a significant role in survival. When evaluated in our series, these variables did not retain significance. Kucera et al. ${ }^{(3)}$ reported that in stage I and II disease, smokers had a decreased survival compared to nonsmokers, but we did not find this in our group of patients. They also reported that diabetes affected survival in stage I and II. Authors have reported that anemic patients have a worsened prognosis and this could not be evaluated in our series. All patients were routinely transfused to maintain hematocrits above 30 , as it was the philosophy that the radiation therapy would be enhanced by this oxygen carrying capacity.

Stage IIA disease of the cervix can be treated with radiation or radical surgery. Our prevailing philosophy during this time period was that radiation could better cover these larger lesions and this was the therapy administered. Thus only one of 27 patients underwent radical hysterectomy. The survival rate for stage IIA reported here is very similar to that reported by Perez et al. ${ }^{(11)}$. We continue to employ radiation therapy as the preferred treatment for stage IIA disease. In this report, the presence of a large stage IIB tumor extending almost to the side wall did not adversely influence survival compared to the smaller stage IIB tumor.

Advanced stage III disease is accompanied almost always by disease to the sidewall. It is rare to have cervical cancer in the lower third of the vagina without extensive central disease extending to the sidewall. In our report, only four patients were stage IIIA, attesting to the rarity of this clinical stage. The $0.4 \%$ reported here is similar to the $1.5 \%$ reported by Cardinale ${ }^{(12)}$. Our survival rate of $50 \%$ in stage IIIA is similar to the $58 \%$ survival rate in 17 patients reported by Cardinale.

The prognosis for squamous cell cancer of the cervix is influenced by the extent of disease and the presence of metastatic disease to regional lymph nodes. The greatest challenge lies in prevention and earlier diagnosis prior to advanced stage disease.

\section{References}

1 Prempree T, Patanaphan V, Sewchand W, Scott RM. The influence of patient's age and tumor grade on the prognosis of carcinoma of the cervix. Cancer 1983; 51: 1764-71.

2 Johnson DW, Cox RS, Billingham G, Ung N, Martinez A. Survival, prognostic factors, and relapse patterns in uterine cervical carcinoma. Am J Clin Oncol 1983; 6: 407-15.

3 Kucera $H$, Enzelsberger $H$, Eppel W, Weghaupt $K$. The influence of nicotine abuse and diabetes mellitus on the results of primary irradiation in the treatment of carcinoma of the cervix. Cancer 1977; 60: 1-4.

4 Kapp DS, Fischer D, Gutierrez E, Kohorn EI, Schwartz PE. Pretreatment prognostic factors in carcinoma of the uterine cervix: a multivariable analysis of the effect of age, stage, histology and blood counts on survival. Int I Radiation Oncology Biol Phys 1983; 9: 445-55.

5 Perez CA, Breaux S, Madoc-Jones H, et al. Radiation therapy alone in the treatment of carcinoma of the uterine cervix. Cancer 1983; 51: 1393-1402.

6 Kaplan EL, Meier P. Non-parametric estimation from incomplete observations. J Am Stat Assoc 1958; 53: 457-81.

7 Mantel N. Evaluation of survival data and two new rank order statistics arising in its consideration. Cancer Chemother Rep 1966; 50: 163-70.

8 Cox DR. Regression models and life tables. J R Stat Soc 1972; 34: 187-202.

9 LaPolla JP, Scheaerth JB, Gaddis O, Morrow CP. The influence of surgical staging on the evaluation and treatment of patients with cervical carcinoma. Gynecol Oncol 1986; 24: 194-206.

10 Stehman FB, Bundy BN, Keys H, Currie JL, Mortel R, Creasman WT. A randomized trial of hydroxyurea versus misonidazole adjunct to radiation therapy in carcinoma of the cervix. Am J Obstet Gynecol 1988; 159: 87-94.

11 Perez CA, Camel M, Kao MS, Askin F. Randomized study of preoperative radiation and surgery or irradiation alone in the treatment of stage IB and IIA carcinoma of the cervix: preliminary analysis of failures and complications. Cancer 1980; 45: $2759-68$.

12 Cardinale JG, Peschel RE, Gutierrez E, Kapp DS, Kohorn EI, Schwartz PE. Stage IIIA carcinoma of the uterine cervix. Gynecol Oncol 1986; 23: 199-204.

Accepted for publication 28 March 1991 